\title{
Phase-correcting non-local means filtering for diffusion-weighted imaging of the spinal cord
}

\author{
Sevgi Gokce Kafali1,2 ｜ Tolga Çukur ${ }^{1,2,3}$ ｜ Emine Ulku Saritas 1,2,3 \\ ${ }^{1}$ Department of Electrical and Electronics Engineering, Bilkent University, Ankara, Turkey \\ ${ }^{2}$ National Magnetic Resonance Research Center (UMRAM), Bilkent University, Ankara, Turkey \\ ${ }^{3}$ Neuroscience Program, Sabuncu Brain Research Center, Bilkent University, Ankara, Turkey
}

\section{Correspondence}

Sevgi Gokce Kafali, Department of Electrical and Electronics Engineering, Bilkent University, Ankara, TR-06800, Turkey.

Email: kafali@ee.bilkent.edu.tr.

\section{Funding information}

European Commission, FP7 Marie Curie

Career Integration Grant, Grant/Award

Number: PCIG13GA618834,

PCIG13GA618101; European Molecular Biology Organization, Installation Grant, Grant/Award Number: 3028; Turkish Academy of Sciences through TUBAGEBIP 2015 program; BAGEP Award of the Science Academy

\begin{abstract}
Purpose: DWI suffers from low SNR when compared to anatomical MRI. To maintain reasonable SNR at relatively high spatial resolution, multiple acquisitions must be averaged. However, subject motion or involuntary physiological motion during diffusion-sensitizing gradients cause phase offsets among acquisitions. When the motion is localized to a small region, these phase offsets become particularly problematic. Complex averaging of acquisitions lead to cancellations from these phase offsets, whereas magnitude averaging results in noise amplification. Here, we propose an improved reconstruction for multi-acquisition DWI that effectively corrects for phase offsets while reducing noise.
\end{abstract}

Theory and Methods: Each acquisition is processed with a refocusing reconstruction for global phase correction and a partial k-space reconstruction via projectiononto-convex-sets (POCS). The proposed reconstruction then embodies a new phasecorrecting non-local means (PC-NLM) filter. PC-NLM is performed on the complexvalued outputs of the POCS algorithm aggregated across acquisitions. The PC-NLM filter leverages the shared structure among multiple acquisitions to simultaneously alleviate nuisance factors including phase offsets and noise.

Results: Extensive simulations and in vivo DWI experiments of the cervical spinal cord are presented. The results demonstrate that the proposed reconstruction improves image quality by mitigating signal loss because of phase offsets and reducing noise. Importantly, these improvements are achieved while preserving the accuracy of apparent diffusion coefficient maps.

Conclusion: An improved reconstruction incorporating a PC-NLM filter for multiacquisition DWI is presented. This reconstruction can be particularly beneficial for high-resolution or high-b-value DWI acquisitions that suffer from low SNR and phase offsets from local motion.

\section{K E Y W O R D S}

denoising, diffusion weighted imaging, motion, non-local means, phase correction, spinal cord
Parts of this work were presented in the Annual Meeting of ISMRM in Singapore, 2016.

\section{1 | INTRODUCTION}

DWI enables the identification of the structure and organization of tissues at the microscopic level. ${ }^{1,2}$ DWI has been widely performed on the brain, but its use in small, deep 
regions such as the spinal cord, prostate, liver, or kidney has been relatively restricted. One of the primary limitations is reduced SNR, ${ }^{1}$ caused by the absence of custom receive-coil arrays with large number of channels that can be placed in close vicinity of these regions (as opposed to 32-96 channels for brain coils). This SNR limitation is especially problematic at high $b$-values and high spatial resolutions. ${ }^{3}$ The spinal cord, for instance, has a relatively small diameter that mandates high-resolution imaging. Moreover, spine array coils typically feature only $6-8$ channels and can only be placed posteriorly to the spine. In such cases, it is common to improve SNR by averaging multiple acquisitions. However, numerous sources of involuntary physiological motion complicate the averaging process. ${ }^{4}$

Motion during diffusion-sensitizing gradients results in $\mathrm{k}$ space shifts and global/local phase offsets among acquisitions. With complex averaging, these shifts/offsets can cause signal cancellations across acquisitions. ${ }^{5}$ The most common approach to this problem in clinical settings is magnitude averaging optionally followed by image denoising. Removing phase information prevents phase-offset related issues, albeit at the expense of lower SNR. ${ }^{6}$ Several studies considered subsequent denoising of magnitude-averaged images to enhance DWI images and ADC estimates. ${ }^{6-21}$ Various denoising methods were proposed for DWI including maximum likelihood (ML) estimation, ${ }^{13}$ linear minimum mean squared error estimation, ${ }^{14-16}$ anisotropic diffusion filtering, ${ }^{17,18}$ transformdomain denoising, ${ }^{21}$ smoothing based on constrained variational principles, ${ }^{19}$ and non-local means (NLM) filtering. ${ }^{9-12,20}$ Recent studies also used the joint information provided by DWI images in different diffusion-encoding directions, with the goal of better preserving the edges that are common across multiple images. $^{7}$ Note that these techniques were demonstrated for DWI of the brain, where higher SNR levels and reduced motion-induced problems are expected compared to the spinal cord. In relatively SNR-starved data sets, however, magnitude averaging can lead to accumulation of noise, which can be difficult to remove via post-processing approaches.

An alternative approach to improve SNR in multipleacquisition DWI is to perform complex averaging after phase correction. This correction can be performed using a refocusing reconstruction ${ }^{22}$ that accounts for global phase errors based on low-resolution phase information. For self-navigated acquisitions (e.g., single-shot acquisitions such as single-shot echo planar imaging (ss-EPI) or multi-shot acquisitions such as PROPELLER ${ }^{23,24}$ ), the low-resolution phase can be extracted from a central portion of the k-space data. Other multi-shot acquisitions, on the other hand, are prone to motion-induced k-space shifts in each interleaf, requiring external navigator echoes ${ }^{25,26}$ or optical motion tracking. ${ }^{27-29}$ However, as we show in this work, correction of phase errors because of bulk motion may not compensate for local phase issues, resulting in local signal cancellations in DWI images.
Here, we propose a reconstruction scheme that incorporates a new phase-correcting non-local means (PC-NLM) filter to mitigate noise and prevent local signal cancellations, while preserving the details of DWI images and yielding accurate ADC estimates. With this technique, we especially target DWI of the spinal cord. We start by demonstrating that the local phase issues in the spinal cord are prominent whenever the diffusion-encoding direction has a significant component along the anterior/posterior $(\mathrm{A} / \mathrm{P})$ direction. Instead of denoising as a post-processing step, the proposed PC-NLM filter is integrated into the reconstruction routine, directly operating on multiple complex-valued acquisitions. We show with extensive simulations and via in vivo high-resolution DWI that the proposed technique suppresses signal cancellations because of local phase errors, while overcoming noise accrual because of intrinsic low-SNR acquisitions.

\section{2 | THEORY}

In this section, we first demonstrate the local phase problems in DWI of the cervical spine, followed by a description of the standard NLM filter. We then explain the proposed PCNLM filter and how it integrates into the overall image reconstruction scheme.

\section{1 | Phase issues in DWI}

As mentioned above, 2 different approaches can be undertaken when combining multiple DWI acquisitions: global phase terms can be corrected using an algorithm such as refocusing reconstruction ${ }^{22}$ before averaging, or phase information can be neglected entirely via magnitude averaging. The shortcomings of both approaches are exemplified in Figure 1. Figure 1A shows the magnitude and phase images of repetitions for DWI of the cervical spine. Despite global phase correction, each repetition displays different patterns of local phases (red arrows). These local phase errors occur at exactly the same position for images obtained from all receive-array channels, indicating that they, in fact, stem from anatomical sources (see Supporting Figure S1). In DWI of the spinal cord, we have observed that the local phase issues are most prominent when the diffusion-encoding direction has a significant component along the A/P direction (see Supporting Figures S2 and S3) and their locations coincide with the nerve roots exiting the spinal cord. This observation indicates a local motion of the cord in the $\mathrm{A} / \mathrm{P}$ direction, which is consistent with the literature on nerve root pulsations in the same direction, synchronized to heart and CSF pulsations. Literature suggests that the local motion of the spinal cord is especially affected by the motion in the radicular arteries because of arterial pulsation and respiration. ${ }^{30-33}$ 


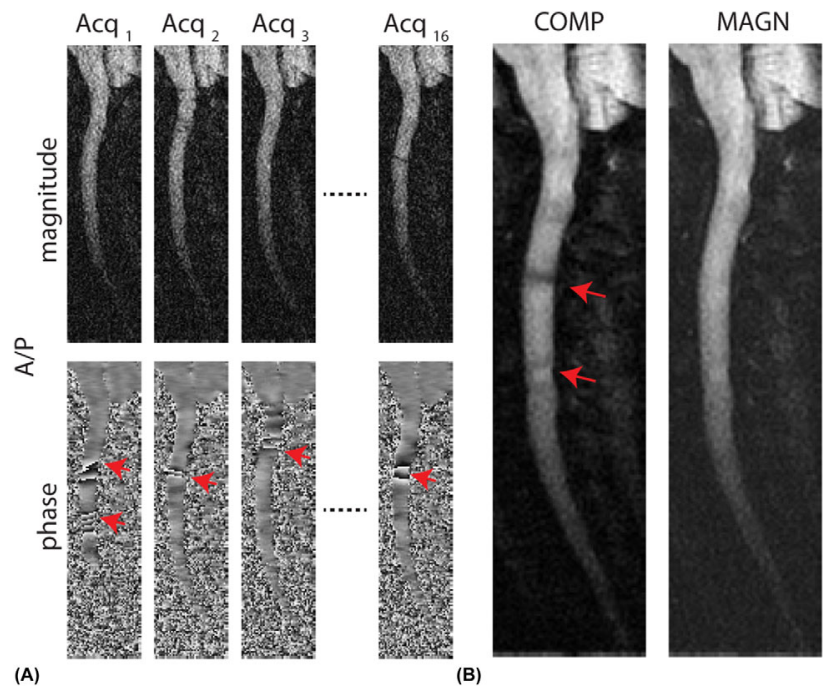

FIG URE 1 Local phase problems in DWI of the cervical spinal cord. (A) Magnitude and phase of each acquisition for a single slice, following a refocusing reconstruction to correct for global phase errors and a partial kspace reconstruction. Local phase errors (shown by red arrows) are especially prominent in the case where diffusion sensitizing gradients are in the $\mathrm{A} / \mathrm{P}$ direction, as in this example. (B) When direct complex averaged, the phase errors result in local signal cancellations. Magnitude averaging, on the other hand, leads to an accumulation of noise especially in regions that were SNR-starved before averaging

As shown in Figure 1B, for complex averaging following global phase correction, local phase errors can result in signal cancellations along the spinal cord. These cancellations can be mistaken with local increases in diffusion. Magnitude averaging, on the other hand, can cause noise amplification in the combined image when each repetition is SNR-starved. The goal of this work is to simultaneously correct for phase errors while boosting the SNR of the combined image.

\section{2 | NLM filtering}

Unlike typical denoising methods that perform filtering across small neighborhoods of pixels, ${ }^{34}$ NLM filtering denoises each pixel by averaging it with other pixels from highly similar patches. The search for similar patches can be performed over the whole image or a specified search area. ${ }^{20}$

Here, we describe the standard 2D NLM filter with the following notations:

- $m\left(x_{i}, y_{i}\right)$ : the central-pixel intensity at location $\left(x_{i}, y_{i}\right)$

- $N_{i}$ : the local 2D neighborhood centered around $\left(x_{i}, y_{i}\right)$, of size $(2 d+1)^{2}, d \in \mathbb{N}$.

- $m\left(N_{i}\right)$ : the neighborhood intensity vector containing the intensities of the pixels in $N_{i}$

- $S_{x}, S_{y}$ : the sizes of the image in $\mathrm{x}$ - and $\mathrm{y}$-directions

- $\Omega^{2}$ : the image grid
- $\left|\Omega^{2}\right|=S_{x}, S_{y}$ : the total number of pixels in the 2D image

- $V_{i}$ : the search volume centered around $\left(x_{i}, y_{i}\right)$, of size $(2 M+1)^{2}, M \in \mathbb{N}$

The filtered intensity is a weighted average of all the pixels in the search area:

$$
m_{N L M}\left(x_{i}, y_{i}\right)=\sum_{\left(x_{j}, y_{j}\right) \in V_{i}} w\left(x_{i}, y_{i} ; x_{j}, y_{j}\right) m\left(x_{j}, y_{j}\right)
$$

Instead of using the distance from the central pixels, the filter weights, $w\left(x_{i}, y_{i} ; x_{j}, y_{j}\right)$, are determined using the similarity between the neighborhood intensity vectors:

$$
w\left(x_{i}, y_{i} ; x_{j}, y_{j}\right)=\frac{1}{Z_{i}} e^{-\frac{\left|m\left(N_{i}\right)-m\left(N_{j}\right)\right|^{2}}{h^{2}}} .
$$

Here, $h \in \mathbb{R}$ is a smoothing parameter calculated according to the noise variance in the image. $Z_{i}$ is a normalization constant, which ensures that the sum of all filter weights is equal to 1 , i.e.,

$$
\sum_{j} w\left(x_{i}, y_{i} ; x_{j}, y_{j}\right)=1
$$

As in all denoising methods, there is a trade-off between the sharpness and SNR of the resulting image. In NLM, this trade-off is controlled via $h$, which needs to be tuned carefully. This parameter can be estimated from the pseudoresiduals as follows ${ }^{35,36}$ :

$$
\boldsymbol{\epsilon}_{i}=\sqrt{\frac{4}{5}}\left|m\left(x_{i}, y_{i}\right)-\frac{1}{4} \sum_{\left(x_{j}, y_{j}\right) \in P_{i}} m\left(x_{j}, y_{j}\right)\right| .
$$

$P_{i}$ is the 4-neighborhood for pixel $\left(x_{i}, y_{i}\right)$ and the constant $\sqrt{4 / 5}$ is used to satisfy $\mathrm{E}\left[\epsilon_{i}{ }^{2}\right]=\sigma^{2}{ }^{20}$ Then the noise variance in the image can be estimated as:

$$
\sigma^{2}=\frac{1}{\left|\Omega^{2}\right|} \sum_{i \in \Omega^{2}} \epsilon_{i}{ }^{2}
$$

Finally, the smoothing parameter can be calculated by:

$$
h^{2}=2 \beta \sigma^{2}\left|N_{i}\right|,
$$

where $\beta \in \mathbb{R}$ is manually tuned between 0.5 and 1 , in correlation with the noise level. ${ }^{20}$

\section{3 | PC-NLM filtering}

In this work, instead of post-processing a reconstructed DWI image via NLM filtering, we incorporate a novel NLM filter into the image reconstruction process to correct for phase errors while boosting SNR. The proposed method relies on the fact that multiple acquisitions (NEX) of the same image share the same underlying structure, but have varying phase 


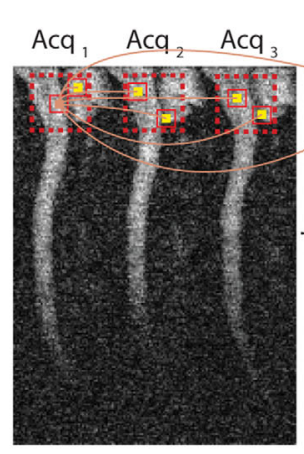

(A)

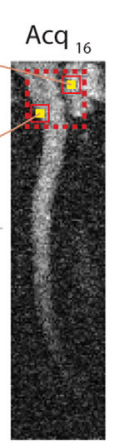

(B)

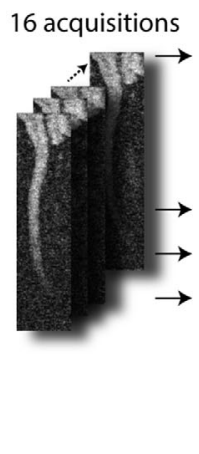

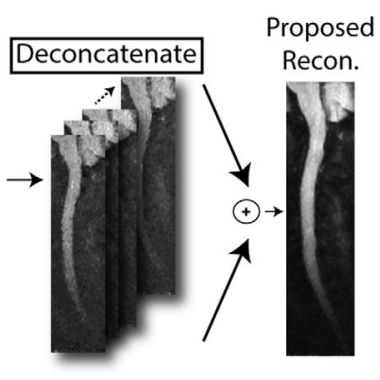

F IG URE 2 The proposed phase-correcting non-local means (PC-NLM) filter and the overall image reconstruction scheme. (A) Multiple image acquisitions are first concatenated to form an aggregate image. The search volume in PC-NLM for finding similar neighborhoods is expanded to include the repeated positions across multiple acquisitions. (B) The proposed reconstruction (illustrated for a single coil): multiple acquisitions are first phasecorrected via a refocusing reconstruction using a central portion of k-space data. After phase correction, individual-acquisition images are processed with the POCS algorithm (3 iterations) for partial k-space reconstruction. The complex-valued outputs of the POCS algorithm are concatenated to form an aggregate image that is processed with the PC-NLM filter. The smoothing parameter of PC-NLM is calculated from concatenated phase-corrected acquisitions to retain sensitivity for detailed image features. The filtered aggregate image is deconcatenated and magnitude averaged across acquisitions

errors and noise. To leverage this property, multiple acquisitions are first concatenated to form a large image of size $S_{x} \times\left(\operatorname{NEX} S_{y}\right)$. This concatenation is performed using complex-valued images. The search volume of NLM is expanded to include patches in identical locations across repeated acquisitions, as demonstrated in Figure 2A. Accordingly, if the original search volume for pixel $\left(x_{i}, y_{i}\right)$ is $V_{i}\left(x_{i}, y_{i}\right)$, the expanded search volume can be expressed as:

$$
V_{i}^{\prime}=\bigcup_{n=1}^{N E X} V_{i}\left(x_{i}, \bmod \left(y_{i}+n S_{y}, \operatorname{NEX} S_{y}\right)\right) .
$$

In the absence of phase errors, the repeated positions in the concatenated image should yield very high similarity to the neighborhood $N_{i}$ of a central pixel. These positions will be effectively complex averaged during the weighted averaging step. In the presence of phase errors, however, the Euclidian distance $\left|m\left(N_{i}\right)-m\left(N_{j}\right)\right|$ will be large because of phase differences among repetitions, reducing the contribution of those positions. In this manner, phase-dependent averaging will suppress signal cancellations stemming from local phase errors.

The primary goal of the PC-NLM filter is to correct for phase errors, while preserving the structural integrity of the reconstructed image. It is also important that the algorithm runs without user intervention. First, to avoid excessive denoising that may result in spatial blurring, we use $\beta=0.5$ in this work. Next, the smoothing parameter is determined automatically using Eq. 6, with a slight modification as explained in the following subsection. Here, the smoothing parameter controls not only the similarity threshold among the neighborhood intensities, but also the tolerable range of phase errors. If the smoothing parameter is set too large, averaging will be performed across many patches, causing spatial blurring. If this parameter is set too small instead, averaging will be performed across only a few patches, compromising noise suppression. Therefore, the smoothing parameter must be set to avoid excessive blurring, while allowing sufficient averaging to take place.

\subsection{Image reconstruction with PC-NLM filtering}

The proposed image reconstruction, outlined in Figure 2B, corrects for global phase issues and reduces the noise level when combining multiple acquisitions. First, multiple acquisitions are phase-corrected using a refocusing reconstruction that estimates the global phase using a fully sampled central portion of k-space data ${ }^{22}$ (ranging between $6-12 \%$ in this work). These individual phase-corrected acquisitions are then separately processed with the projection-onto-convex-sets (POCS) algorithm for partial k-space reconstruction with 3 iterations. ${ }^{37}$ The complex-valued outputs of the POCS algorithm are concatenated across acquisitions to form an aggregate image, which is then processed with the PC-NLM filter. The neighborhood size and search volume are set to $d=1$ and $M=5$, as these values were previously shown to yield the lowest RMS error between ideal noise-free images and NLM-filtered noisy images. ${ }^{20}$ Finally, the output of the PCNLM filter is deconcatenated, and the resulting images are magnitude averaged across acquisitions. The proposed reconstruction is performed individually for each coil, and the coil images are combined via square-root of sum-of-squares.

For a conventional NLM filter, the smoothing parameter would be calculated using the image that is input to the filter (i.e., the aggregate image at the output of the POCS algorithm in this case). However, POCS amplifies high-frequency noise while filling unacquired $\mathrm{k}$-space and this can lead to 
overestimation of the smoothing parameter that depends on the estimated noise. Here, the smoothing parameter is instead estimated directly on global-phase-corrected acquisitions concatenated across acquisitions (see Figure 2B). This estimation procedure increases sensitivity for detailed image features in the subsequent PC-NLM step.

\section{3 | METHODS}

\section{1 | DWI simulations}

Simulations were carried out using the spinal cord MRI data extracted from PropSeg Spinal Cord Segmentation Toolbox. ${ }^{38,39}$ A sample $T_{2}$-weighted sagittal image provided in the toolbox is shown in Figure 3A (original FOV $=264 \times$ $384 \mathrm{~mm}^{2}, \mathrm{TE}=119 \mathrm{~ms}, \mathrm{TR}=1500 \mathrm{~ms}, 1 \times 1 \mathrm{~mm}^{2}$ inplane resolution). The original FOV was reduced to a smaller FOV of $110 \times 54 \mathrm{~mm}^{2}$ to approximate the in vivo images acquired in this work. PropSeg Toolbox was used to obtain segmented tissue masks of CSF, gray matter (GM), and white matter (WM). The other tissues were marked as "others" in this work. These masks were corrected using dilation/erosion operations, and spatially smooth transitions between tissues were achieved via frequency-domain apodization of the masks. The purpose of these corrections was to obtain simulated diffusion-weighted images that match the in vivo images, with soft transitions between tissue boundaries. The final tissue segmentations are displayed in Figure 3B.

Next, a diffusion-weighted image was simulated based on the $\mathrm{T}_{2}$-weighted image. The mean $\mathrm{ADC}$ for $\mathrm{GM}$ and WM were taken as $900 \times 10^{-6} \mathrm{~mm}^{2} / \mathrm{s}$ and $700 \times 10^{-6}$ $\mathrm{mm}^{2} / \mathrm{s}$, respectively. ${ }^{4,40}$ The reported mean ADC values for CSF vary between $4600 \times 10^{-6} \mathrm{~mm}^{2} / \mathrm{s}$ and $8000 \times 10^{-6}$ $\mathrm{mm}^{2} / \mathrm{s}^{4,41}$ Similarly, the in vivo experiments in this work yielded ADC values between $5500 \times 10^{-6} \mathrm{~mm}^{2} / \mathrm{s}$ and $7500 \times 10^{-6} \mathrm{~mm}^{2} / \mathrm{s}$ for CSF. Note that these ADC values for spinal CSF are biased by pulsation effects, as they are larger than free water diffusivity at body temperature. Here, we have chosen $7000 \times 10^{-6} \mathrm{~mm}^{2} / \mathrm{s}$ for the ADC of CSF to match the in vivo diffusion-weighted images where CSF regions are devoid of signal. The tissues labeled as "others" were assumed to consist of muscle with an ADC of $1500 \times 10^{-6} \mathrm{~mm}^{2} / \mathrm{s}$. To match the in vivo experiments, $b=500 \mathrm{~s} / \mathrm{mm}^{2}$ was assumed. The resulting DWI image is shown in Figure 3C.

Next, NEX $=16$ acquisitions were simulated for the DWI image, each with zero initial phase. These acquisitions were then processed to induce similar k-space shift and phase errors as those observed in the in vivo experiments. Accordingly, a linear global-phase term was added in the image domain to model $\mathrm{k}$-space shifts because of bulk motion during diffusion-sensitizing gradients. The k-space of each acquisition was shifted in $\mathrm{k}_{\mathrm{x}}$ and $\mathrm{k}_{\mathrm{y}}$ directions, with the

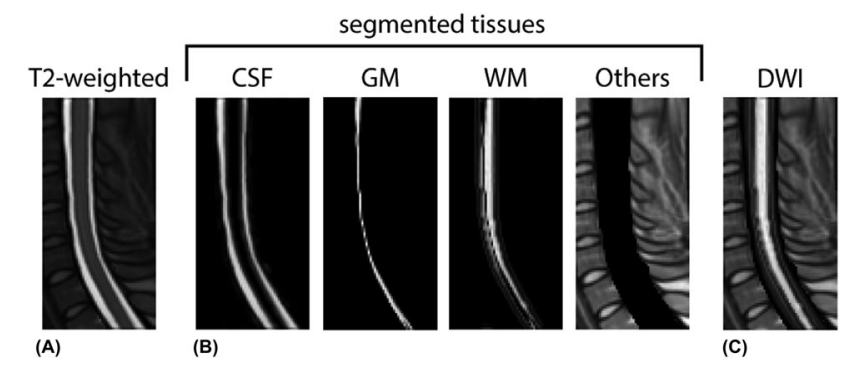

FIGURE 3 Process for simulating a diffusion-weighted image based on a $\mathrm{T}_{2}$-weighted input image. (A) A sample $\mathrm{T}_{2}$-weighted sagittal image from PropSeg Spinal Cord Segmentation Toolbox. (B) Segmented tissues: cerebrospinal fluid (CSF), gray matter (GM), white matter (WM), and "others." (C) The resultant DWI image for $b=500 \mathrm{~s} / \mathrm{mm}^{2}$ is depicted without any noise or phase added

amount of shift randomly picked from a uniform distribution between $[-0.2,0.8]$ pixels. A $2 \mathrm{D}$ local phase term was then added to each image to model the phase errors because of local motion dominant in the $\mathrm{A} / \mathrm{P}$ direction. These local phases, $\phi(\mathrm{x}, \mathrm{y})$, were added to locations near the nerve roots where the local motion was reported to be the most problematic $^{31}$ and were calculated as follows ${ }^{5}$ :

$$
\phi(\mathrm{x}, \mathrm{y})=\gamma G \delta \Delta(\mathrm{x}, \mathrm{y}) .
$$

Here, $\mathrm{x}$-direction is superior-inferior $(\mathrm{S} / \mathrm{I})$ direction, $\mathrm{y}$ direction is the A/P direction, $\gamma$ is the gyromagnetic ratio, $G$ is the amplitude of the diffusion-sensitizing gradient (assumed to be in the $\mathrm{A} / \mathrm{P}$ direction), $\delta$ is the duration of the diffusion-sensitizing gradient, and $\Delta(\mathrm{x}, \mathrm{y})$ is the level of the motion in the $\mathrm{A} / \mathrm{P}$ direction. Here, $\Delta(\mathrm{x}, \mathrm{y})$ was modeled as a zero-mean unit-amplitude Gaussian window in the $\mathrm{x}$-direction, and a Hanning-tapered window in the $y$-direction, affecting a region of size $36 \times 16$ pixels, i.e.,

$$
\Delta(\mathrm{x}, \mathrm{y})=\Delta_{A} \mathrm{~W}_{\text {Gauss }}(\mathrm{x}) \mathrm{W}_{\text {Hanning }}(\mathrm{y}) .
$$

The SD of the Gaussian window was randomly picked from a uniform distribution between $[0.7,1.1]$ pixels for each acquisition, while the Hanning-tapered window was kept the same with a 4-pixel wide transition region on both sides and an 8-pixel wide flat region. The peak motion amplitude $\Delta_{A}$ was chosen from a uniform distribution between $[0.2,0.4] \mathrm{mm}$, in accordance with the ones reported in the literature for the cervical spinal cord. ${ }^{30,32}$

Finally, complex-valued Gaussian noise was added to the $\mathrm{k}$-space data of each acquisition at 13 different noise levels. The noise-to-signal ratio (NSR) of the images varied linearly between $[0,0.750]$, where the SD of noise was used to define the noise level (chosen to be identical for real and imaginary components) and the peak image intensity was used to define the signal level. Equivalently, using SNR $=1 / \mathrm{NSR}$, the SNR of each acquisition ranged between infinity and 1.33. To match in vivo experiments, a $62.5 \% \mathrm{k}$-space coverage was used for each acquisition (i.e., $37.5 \%$ of $\mathrm{k}$-space data were replaced with zeros in $\mathrm{k}_{\mathrm{y}}$ direction). 


\section{2 | In vivo DWI experiments}

In vivo DWI images of the cervical spinal cord of healthy subjects were acquired in the sagittal plane on a $3 \mathrm{~T}$ GE MR 750 scanner, in accordance with the institutional review board protocol. Among the subjects imaged, 2 cases that demonstrated the signal cancellation issues were chosen prospectively.

For the first subject, a 6-channel cervico-thoracic-lumbar (CTL) coil was used. To achieve high in-plane resolution, a reduced-FOV acquisition in the phase-encode (PE) direction was implemented via a 2D-selective RF excitation pulse. ${ }^{42}$ ss-EPI readout with $192 \times 48$ imaging matrix and $62.5 \%$ partial k-space coverage in the PE direction was used. Diffusion weighting was applied in 3 orthogonal directions (S/I, $\mathrm{A} / \mathrm{P}, \mathrm{R} / \mathrm{L}$ ) with $b=500 \mathrm{~s} / \mathrm{mm}^{2}$, a reasonable value for the spinal cord in low-SNR cases. ${ }^{43}$ Other imaging parameters were: $0.94 \times 0.94 \mathrm{~mm}^{2}$ in-plane resolution, $\mathrm{FOV}=18 \times$ $4.5 \mathrm{~cm}^{2}, 4 \mathrm{~mm}$ slice thickness, 6 slices, $\mathrm{TE}=51.3 \mathrm{~ms}$, $\mathrm{TR}=3600 \mathrm{~ms}, \mathrm{NEX}=16$ averages, and a total scan time of 3 min $52 \mathrm{~s}$. For the second subject, an 8-channel CTL coil was used. DTI images with 12 directions at $b=500 \mathrm{~s} / \mathrm{mm}^{2}$ were acquired, along with $2 \mathrm{~T}_{2}$-weighted images. The imaging parameters were: $1 \times 1 \mathrm{~mm}^{2}$ in-plane resolution, $\mathrm{FOV}=20 \times 5 \mathrm{~cm}^{2}, 3 \mathrm{~mm}$ slice thickness, 6 slices, TE $=$ $52 \mathrm{~ms}, \mathrm{TR}=4600 \mathrm{~ms}, \mathrm{NEX}=16$, and a total scan time of $17 \mathrm{~min}$. Other imaging parameters were kept the same as in the first subject.

\section{3 | Alternative reconstructions and comparison of image quality}

Five alternative reconstructions were implemented for comparison. In common with the proposed reconstruction, all alternative reconstructions processed each of the multiple acquisitions with a refocusing reconstruction, ${ }^{22}$ followed by the POCS partial k-space reconstruction. ${ }^{37}$ Each reconstruction then implemented a unique processing on outputs of the POCS algorithm:

1. Complex averaging (COMP): multiple acquisitions were complex averaged.

2. NLM-filtered complex averaging: (NLM-COMP): each acquisition was NLM filtered individually. The smoothing parameter was calculated immediately before filtering, as in the standard NLM filter. ${ }^{20}$ As in PC-NLM, $d=1$ and $M=5$ were used. Finally, the NLM-filtered acquisitions were complex averaged.

3. Magnitude averaging (MAGN): multiple acquisitions were magnitude averaged.

4. NLM-filtered magnitude averaging: (NLM-MAGN): the magnitude image of each acquisition was NLM filtered individually. The smoothing parameter was calculated immediately before filtering, based on the magnitude image. Again, $d=1$ and $M=5$ were used. Finally, the NLM-filtered acquisitions were magnitude averaged.

5. Model fitting (MOD-FIT): magnitude images from multiple acquisitions were used to fit a Rician noise distribution model to each pixel. ${ }^{44}$ Model fitting was carried out via ML estimation using 2 independent variables of the Rice distribution, $\sigma_{g}$ and $\eta .{ }^{45}$ Here, $\sigma_{g}$ is the SD of the underlying complex Gaussian noise (identical for real and imaginary channels) and $\eta$ is the true signal intensity for the diffusion-weighted image in the absence of noise. A typical strategy is to calculate $\sigma_{g}$ from the background of an MRI image. ${ }^{46}$ Because of lack of a background region in reduced-FOV images, here, we performed the following: first, an initial model fitting was carried out for each pixel location individually, using the intensities from NEX $=16$ repetitions. From the resulting map of $\sigma_{g}$ values, the mean $\sigma_{g}$ across all pixel locations was calculated. Finally, this mean $\sigma_{g}$ value was enforced via a constrained model fit that only calculated $\eta$ for each pixel. For pixels where a non-negative $\eta$ cannot be fit, a small pixel intensity of $\sigma_{g} / 100$ was assigned. Assuming that the underlying noise level is constant throughout the image, the intermediate step of calculating the mean $\sigma_{g}$ significantly improves model fitting results, especially under high noise levels.

To assess the reconstructions in terms of image quality and the accuracy of the computed ADCs, a reference DWI image, $\mathrm{DWI}_{\mathrm{ref}}$, was produced without noise or phase errors. For unbiased comparison, a $62.5 \%$ k-space coverage was assumed for the reference image, processed with the refocusing reconstruction ${ }^{22}$ and the POCS partial $\mathrm{k}$-space reconstruction. ${ }^{37}$ Reference $\mathrm{ADC}$ map, $\mathrm{ADC}_{\mathrm{ref}}$, was generated

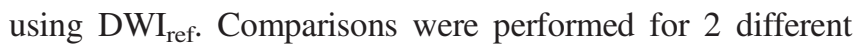
scenarios: (1) when only global phase errors are present to test potential image deterioration when diffusion encoding is in non-problematic directions, and (2) when both global and local phase errors are present.

To quantitatively assess image quality, the peak-signalto-noise ratio (PSNR) metric was calculated:

$$
P S N R=10 \log _{10} \frac{\operatorname{MAX}\left(D W I_{\text {out }}\right)^{2}}{\frac{1}{\left|\Omega^{2}\right|} \sum_{i \in \Omega^{2}}\left[D W I_{\text {ref }}\left(x_{i}, y_{i}\right)-D W I_{\text {out }}\left(x_{i}, y_{i}\right)\right]^{2}},
$$

where $\mathrm{DWI}_{\text {out }}$ is the output DWI image for a given method. To judge perceptual image quality, a secondary assessment was performed using the structural similarity (SSIM) index. ${ }^{47}$ The built-in SSIM function of MATLAB 2016b (The MathWorks, Natick, MA) was used with the default parameters of $\mathrm{C}_{1}=1 \times 10^{-4}$ and $\mathrm{C}_{2}=9 \times 10^{-4}$, where the dynamic 
range of the pixel intensities was between [0, 0.25]. For image quality assessments, Monte Carlo simulations were performed via repeating the simulations 10 times at 13 different noise levels ranging between NSR $=0$ to NSR $=0.750$. The PSNR and SSIM values were averaged across repeats. Note that image quality assessments were not performed for in vivo data sets, because it was not possible to generate reference DWI images.

Last, to analyze the noise statistics in different regions of the resulting images, a separate Monte Carlo simulation was performed with 40 repetitions at a noise level of NSR $=0.25$. For each repetition, the local and global phases were kept identical, and only the noise was randomly generated. For each method, the SDs across 40 repetitions were computed at each pixel location of the output DWI images.

\section{4 | RESULTS}

\section{1 | DWI simulation results}

The magnitude and phase of each acquisition for the simulated dataset at NSR $=0.250$ for the case of both global and local phase errors are shown in Supporting Figure S4C. Accordingly, random local phase was added to 2 different locations along the length of the spinal cord (similar to the in vivo case illustrated in Figure 1A). Images from the proposed and alternative reconstructions at 3 selected noise levels between NSR $=[0.125,0.500]$ in Figure 4, with identical gray-scale windowing. Here, NSR $=0.250$ yielded DWI images that were visually the most similar to the in vivo results (see Supporting Figure S4 for a closer look at results at this noise level for the cases with global phase only and global and local phase errors).

In Figure 4, regardless of the noise level, COMP that performs direct complex averaging results in signal cancellations. On the other hand, MAGN that performs magnitude averaging results in relatively poor SNR because of noise amplification, causing a washed-out appearance especially at higher noise levels. Although NLM-COMP and NLM-MAGN have visibly improved SNR because of filtering, NLM filtering on individual acquisitions does not address the signal cancellation or background noise amplification problems. MOD-FIT results in an unnatural look with a darkening of background at increasing noise levels, because of the difficulty of model fitting in low SNR regions. In contrast, the proposed reconstruction renders a combined image with visibly enhanced SNR without introducing signal cancellations.

Image quality with the proposed and alternative reconstructions was assessed via the PSNR metric. The simulations were repeated 10 times at 13 different noise levels between NSR $=[0,0.750]$, and the PSNR values were averaged across repeats. Figure 5A shows the PSNR values in the presence of global phase errors only. PSNR values for

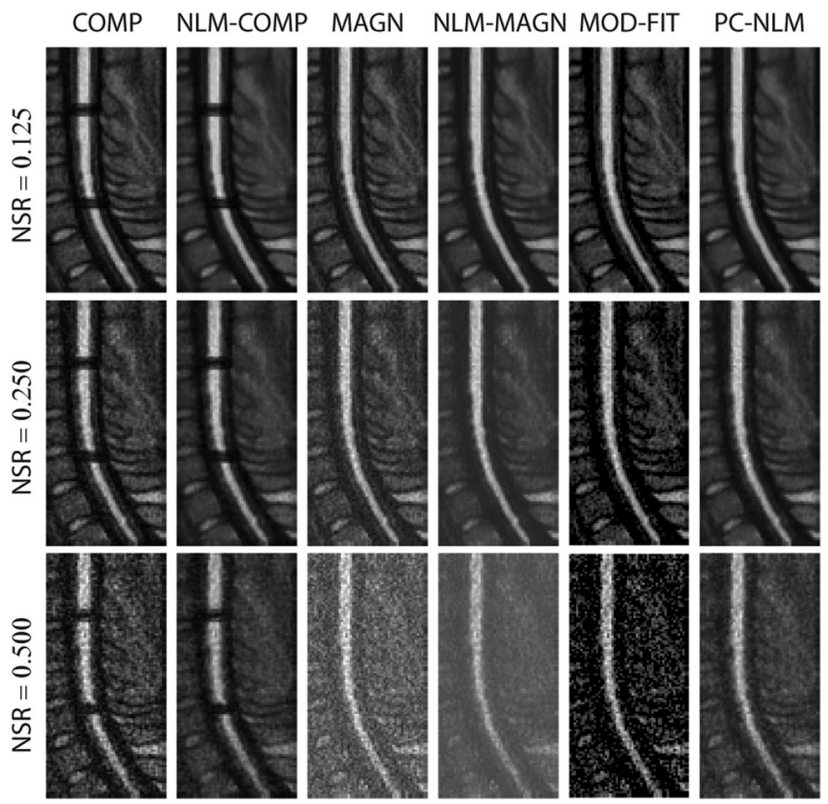

FIGURE 4 DWI simulation results at three different NSR levels $(0.125,0.250$, and 0.500$)$. Reconstruction outputs of the proposed method and alternative methods are displayed for NEX $=16$. COMP and NLMCOMP show signal cancellations because of phase errors regardless of the noise level. On the other hand, MAGN and NLM-MAGN yields pixel intensities that are comparable to the noise floor, especially at higher noise levels. MOD-FIT is sensitive to noise and yields an unnatural look as the noise level increases. The proposed reconstruction achieves superior noise suppression compared to methods based on magnitude averaging and model fitting, and alleviates signal cancellations compared to methods based on complex averaging

COMP and NLM-COMP are in 30-45 dB range at all noise levels, and higher than PSNR values for MAGN, NLMMAGN, and MOD-FIT when NSR $>0.150$. (Note that MOD-FIT shows degradation in image quality starting as early as NSR $>0.100$.) This trend is expected, as robustness against noise amplification is the main advantage of complex averaging. Meanwhile, there are some subtle differences in quality introduced by NLM filtering for complex averaging and magnitude reconstructions (i.e., between NLM-COMP and COMP and between NLM-MAGN and MAGN). Whereas NLM filtering slightly reduces PSNR at relatively low noise levels, it yields a minor increase in PSNR at higher noise levels. These results suggest that NLM causes smoothing of detailed image features to suppress noise, and therefore its advantages are more apparent at higher noise levels. The proposed reconstruction maintains a favorable trade-off between NLM-COMP and NLM-MAGN at very low and high noise levels (NSR $<0.1$ and NSR $>0.4$ ), and superior performance compared to all other techniques in a broad range of realistic noise levels $(0.1<\mathrm{NSR}<0.4)$. At $\mathrm{NSR}=0.250$, the proposed method has comparable PSNR to COMP (38.3 dB vs. $38.2 \mathrm{~dB}$ ), $4 \mathrm{~dB}$ higher PSNR than MAGN (38.3 dB vs. $34.3 \mathrm{~dB}$ ), and $6.9 \mathrm{~dB}$ higher PSNR than MOD-FIT (38.3 dB vs. $31.4 \mathrm{~dB}$ ). 
Global Phase Only

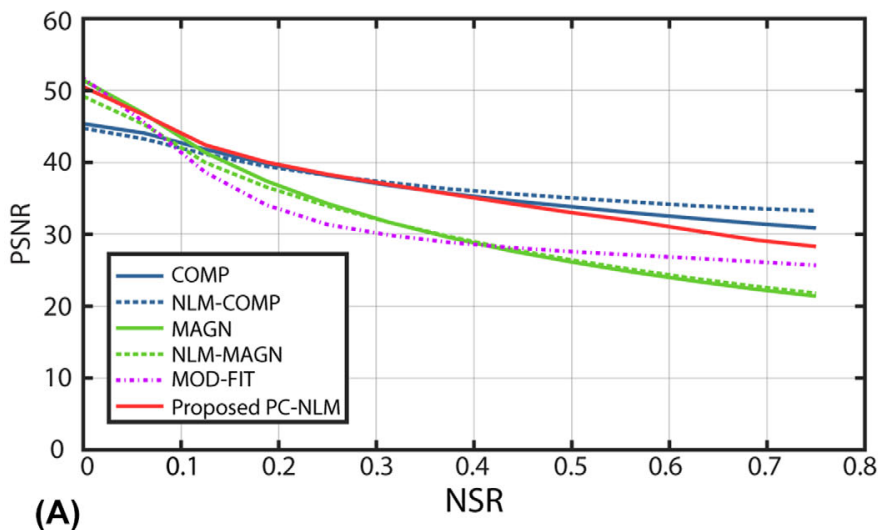

(A)

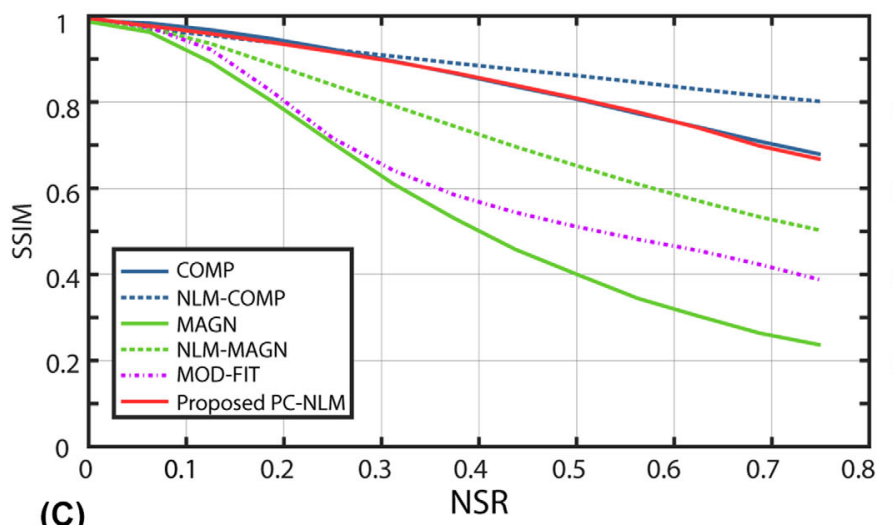

(C)

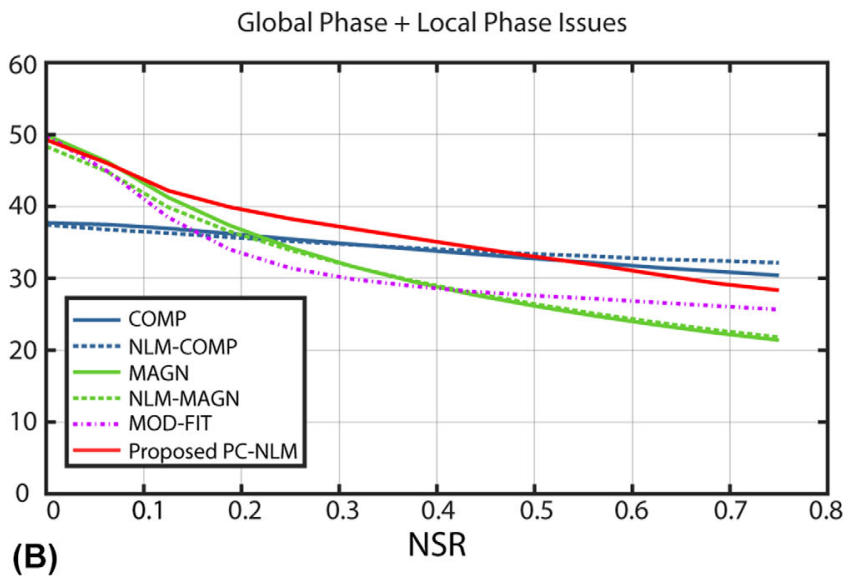

(B)

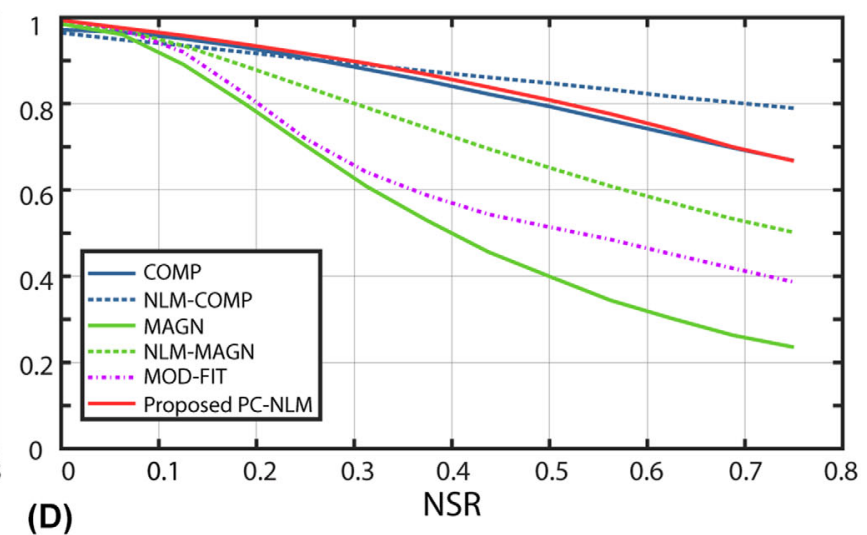

F I G URE 5 Image quality assessment for the proposed and alternative reconstructions based on PSNR and SSIM metrics. The proposed reconstruction achieves a high level of image quality across a broad range of realistic noise levels (NSR $<0.400$ ). (A) In the presence of only global phase errors, PSNR values for COMP and NLM-COMP remain at high levels for all NSR values. Meanwhile, PSNR for MAGN, NLM-MAGN, and MOD-FIT drop immediately with increasing noise. (B) When local phase errors are also incorporated, PSNR for COMP and NLM-COMP decrease $\sim 1-7 \mathrm{~dB}$ because of local signal cancellations. The trends in MAGN, NLM-MAGN, and MOD-FIT remain the same. The proposed reconstruction shows PSNR values that converge MAGN at low noise levels and that converge COMP at high noise levels around NSR $\approx 0.500$. This result suggests that PC-NLM captures the phase robustness of MAGN and noise robustness of COMP simultaneously. (C and D) SSIM values of COMP and NLM-COMP do not change significantly between the two scenarios (i.e., global phase errors vs. both global and local phase errors). Therefore, SSIM is not sufficiently sensitive to capture phase-induced signal cancellation. SSIMs for MAGN and NLM-MAGN drop significantly with increasing NSR because of noise accumulation. SSIM for MOD-FIT drops similarly at higher NSR because of noise sensitivity. Last, the proposed reconstruction maintains the highest SSIM values for NSR $<0.300$, and comparable SSIM to COMP at higher noise levels

Figure 5B shows the PSNR values in the presence of both global and local phase errors. Compared to the simulations with only global phase errors, PSNR values drop $\sim 1.0-7.0 \mathrm{~dB}$ for COMP and NLM-COMP because of local signal cancellations. Meanwhile, PSNR values for MAGN, NLM-MAGN, and MOD-FIT are not affected. Still, MAGN and MOD-FIT yield better PSNR performance at low noise levels, and COMP yields better PSNR performance at high noise levels. Note that the proposed technique achieves PSNR similar to MAGN at low noise and COMP at high noise, capturing the phase robustness of MAGN and noise robustness of COMP simultaneously. When compared to Figure 5A, the PSNR values of the proposed PC-NLM method are only reduced by $0.5-1.0 \mathrm{~dB}$ for NSR $<0.100$, and this difference reduces to $<0.1 \mathrm{~dB}$ at higher noise levels. At NSR $=0.250$, the proposed method has $2.8 \mathrm{~dB}$ higher
PSNR than COMP (38.3 dB vs. $35.5 \mathrm{~dB}), 4 \mathrm{~dB}$ higher PSNR than MAGN (38.3 dB vs. $34.3 \mathrm{~dB}$ ) and $6.9 \mathrm{~dB}$ higher PSNR than MOD-FIT (38.3 dB and $31.4 \mathrm{~dB}$ ). At a higher noise level of NSR $=0.500$, PC-NLM has $6.6 \mathrm{~dB}$ higher PSNR than NLM-MAGN (33.0 dB vs. $26.4 \mathrm{~dB}$ ), $5.4 \mathrm{~dB}$ higher than MOD-FIT (33.0 dB vs. $27.6 \mathrm{~dB}$ ), and comparable PSNR to NLM-COMP (33.0 dB vs. $33.4 \mathrm{~dB}$ ). It is important to note that although COMP and NLM-COMP yield the highest PSNR at high noise levels, they both suffer from signal cancellation. An aggregate PSNR metric over the entire image can be ineffective in capturing these local artifacts.

To assess the perceptual quality of DWI images that also reflect image sharpness, a secondary assessment was performed using the SSIM metric. Figures 5C and 5D show the SSIM values in the presence of only global phase errors and in the presence of both global and local phase errors, 


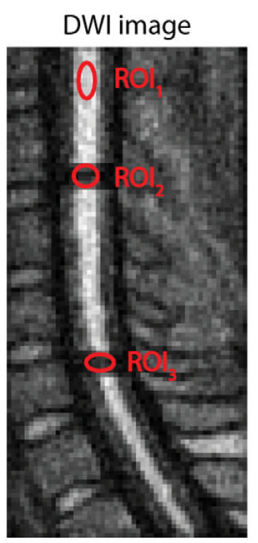

(A)
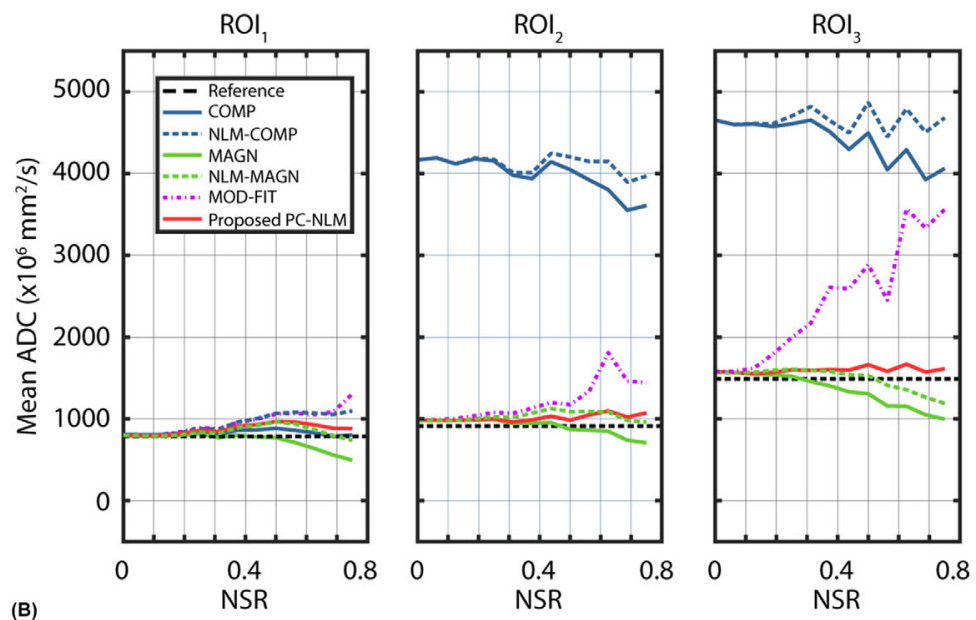

F I G URE 6 Comparison of ADC values with respect to the reference ADC map. (A) Three ROIs are selected to analyze the effects of noise accumulation and phase-related signal cancellations. $\mathrm{ROI}_{2}$ and $\mathrm{ROI}_{3}$ have phase issues, whereas $\mathrm{ROI}_{1}$ does not. $\mathrm{ROI}_{3}$ also has reduced $\mathrm{SNR}$. (B) $\mathrm{COMP}$ and NLM-COMP yield overestimated results in $\mathrm{ROI}_{1}$ as noise increases. Signal cancellations in COMP and NLM-COMP lead to overestimation of the ADCs in ROI ${ }_{2}$ and $\mathrm{ROI}_{3}$, whereas noise accumulation in MAGN and NLM-MAGN result in underestimation of the ADCs especially in ROI 3 . MOD-FIT overestimates the ADC values at higher noise levels, especially in $\mathrm{ROI}_{2}$ and $\mathrm{ROI}_{3}$. The proposed reconstruction is robust against noise in the $\mathrm{ADC}$ estimations in all $3 \mathrm{ROIs}$

respectively. Overall, SSIM values for all reconstruction methods tested remain similar between the two scenarios depicted in Figures 5C and 5D. SSIM values for COMP and NLM-COMP are higher than those for MAGN, NLMMAGN, and MOD-FIT when NSR $>0.100$. Compared to PSNR, the quality improvement with NLM filtering at high noise levels is more clearly captured in SSIM measurements. For instance, SSIM for MAGN drop from 0.99 to 0.24 as the noise level is increased, whereas SSIM for NLM-MAGN only drops from 0.99 to 0.51 in the same range. The SSIM for MOD-FIT also drops significantly from 0.99 to 0.34 at increasing noise levels. Last, the proposed reconstruction maintains the highest SSIM values for NSR $<0.300$, and comparable SSIM to COMP at higher noise levels. Again, it should be noted that both COMP and NLM-COMP suffer from signal cancellations despite their high SSIM performances. Similar to PSNR, SSIM is an aggregate metric across the entire image that is insufficiently sensitive in detecting local artifacts.

For the simulated datasets, ADC maps were also compared with $\mathrm{ADC}_{\text {ref. }}$. Figure 6 shows the mean $\mathrm{ADC}$ values in 3 different regions of interest (ROIs), selected as follows:

1. A region with no phase issues and relatively high SNR (because of high local coil sensitivities),

2. a region with local phase issues, and

3. a region with local phase issues and relatively low SNR (because of low local coil sensitivities).

The plots in Figure 6 show the average ADC values within each ROI across 10 Monte Carlo simulations. With respect to $\mathrm{ADC}_{\text {ref }}$ in $\mathrm{ROI}_{1}, \mathrm{COMP}$, NLM-COMP, MOD-FIT, and the proposed reconstruction tend to slightly overestimate the ADC as noise increases. MAGN, on the other hand, underestimates the ADC at high noise levels because of noise amplification. NLM-MAGN also displays a similar trend, but only at very high noise levels. In $\mathrm{ROI}_{2}$ and $\mathrm{ROI}_{3}$, signal cancellations in COMP and NLM-COMP lead to significant overestimation of ADC values, whereas noise amplification in MAGN and NLM-MAGN result in underestimation of ADC especially in $\mathrm{ROI}_{3}$. MOD-FIT overestimates the ADC values even at realistic noise levels around NSR $=0.250$, especially in $\mathrm{ROI}_{3}$. This problem quickly escalates at higher noise levels because of the difficulty of model fitting in low-SNR cases. The proposed reconstruction yields ADC values that closely match the reference ADCs in all three ROIs, with a relatively minor tendency to overestimate. It is important to note that although the error in the ADC is overall comparable for the proposed method and MAGN/NLM-MAGN, the proposed method achieves superior noise suppression and significantly higher PSNR/ SSIM results when compared to those 2 methods.

Last, the noise statistics were analyzed via a Monte Carlo simulation with 40 repetitions at NSR $=0.250$. Global and local phases were kept identical among repetitions to investigate their effects on local noise characteristics. Figure 7 shows the SD of pixel intensities across 40 repetitions for each reconstruction technique (see Supporting Table S1 for values in 3 different ROIs). COMP shows high SD throughout the spinal cord, except for regions of local signal cancellations where SD is lower because of lower pixel intensities. MAGN shows uniform SD throughout the spinal cord, with SD levels matching that of COMP except in areas of local signal cancellations. As expected, NLM-COMP and NLMMAGN have relatively lower SD than their non-filtered counterparts. Furthermore, NLM filtering yields spatially 


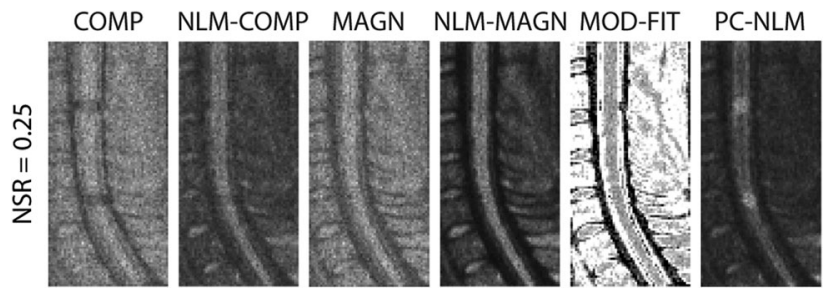

F IG URE 7 Local noise statistics for the proposed and alternative reconstructions at NSR $=0.250$. COMP and MAGN result in comparable high SD along the spinal cord, except in regions of signal cancellation where SD for COMP decreases. NLM-COMP and NLM-MAGN have reduced SD when compared to COMP and MAGN. MOD-FIT gives the highest SD across all the reconstruction techniques, because of its noise sensitivity. PC-NLM yields the lowest SD in regions without signal cancellations. In the problematic regions, SD of PC-NLM converges to that of MAGN. See Supporting Table S1 for SD values in 3 different ROIs

varying noise based on the signal level. MOD-FIT results in the highest SD across all techniques, suffering especially in the background regions with lower signal intensities. In contrast, the proposed PC-NLM yields the lowest SD in regions without local signal cancellations. Even in cancellation regions, the SD for PC-NLM does not exceed that of MAGN. This can be attributed to the fact that, in the presence of excessive local phase variations, PC-NLM cannot find a similar patch in other NEXs and therefore leaves the central pixel unaveraged. Because filtered NEXs are then magnitude averaged, the noise characteristics would then match that of MAGN in this worst case. For noise statistics in the presence of global phase only, see Supporting Figure S5 and Supporting Table S1.

\section{2 | In vivo experimental results}

Figures $8 \mathrm{~A}$ and $8 \mathrm{~B}$ display the results in the first subject, for single-channel and combined-channel DWI images with diffusion-weighting in the $\mathrm{A} / \mathrm{P}$ direction. The red arrows indicate regions of signal cancellation in COMP and NLMCOMP reconstructions. The blue arrows indicate regions of noise amplification in MAGN, NLM-MAGN, and MOD-FIT reconstructions, prominent in the inferior parts of the spinal cord because of reduced local coil sensitivity. In contrast, the proposed reconstruction suppresses signal cancellations and noise amplification and ensures superior depiction of the spinal cord even in the inferior regions. As a trade-off of noise suppression, there is a slight loss of resolution in NLMCOMP, NLM-MAGN, and PC-NLM (e.g., visible in the cerebellum region in Figure 8). This effect is less pronounced for the proposed method, thanks to the automated tuning of the smoothing parameter.

The mean ADC values in the spinal cord were analyzed by selecting 3 different ROIs (see red dashed circles in Figure $8 \mathrm{~B})$ :
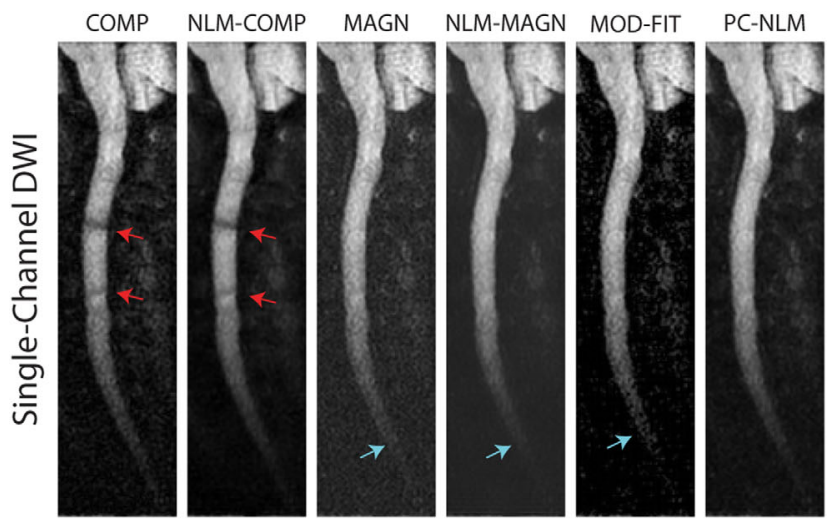

(A)


(B)

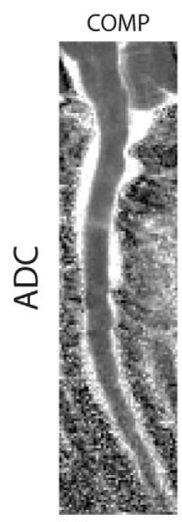

NLM-COMP
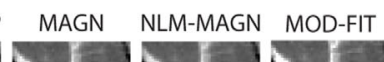

PC-NLM

(C)
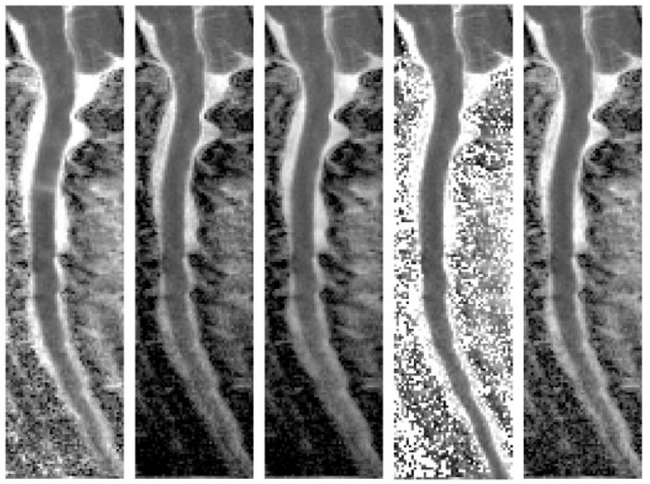

FIG URE 8 Results for in vivo DWI of the spinal cord for the first subject. Images with diffusion-encoding along the A/P direction are displayed. (A) Reconstructions of single-channel images. Local signal cancellations because of phase errors are prominent in COMP and NLMCOMP (red arrows). MAGN, NLM-MAGN, and MOD-FIT overcome signal cancellations, but noise accrual or noise sensitivity occurs (blue arrows). (B) Reconstructions of combined-channel images. Signal cancellations (red arrows) are still present in COMP and NLM-COMP. Although combining across channels improves depiction of the inferior parts of the spinal cord, noise amplification remains a problem in MAGN and NLMMAGN. Although MOD-FIT also benefits from combining across channels, the unnatural darkening in the background persists. In contrast, the proposed reconstruction suppresses signal cancellations and noise amplifications. For the quantitative analyses of the mean ADC values, 3 different ROIs were selected, as marked on the DWI image from COMP. (C) ADC maps for all reconstructions. The mean ADC values in the selected ROIs are listed in Table 1 
TABLE 1 Mean and SD of ADC values

\begin{tabular}{llll} 
& \multicolumn{3}{l}{$\begin{array}{l}\text { ADC } \\
\left(\times \mathbf{1 0}^{-\mathbf{6}} \mathbf{~ m m}^{2} / \mathbf{s}\right)\end{array}$} \\
\cline { 2 - 4 } & $\mathbf{R O I}$ & $\mathbf{R O I}_{\mathbf{2}}$ & $\mathbf{R O I}_{\mathbf{3}}$ \\
\hline COMP & $696 \pm 191$ & $1549 \pm 432$ & $742 \pm 240$ \\
\hline NLM-COMP & $699 \pm 160$ & $1557 \pm 420$ & $841 \pm 223$ \\
\hline MAGN & $668 \pm 189$ & $549 \pm 255$ & $613 \pm 243$ \\
\hline NLM-MAGN & $671 \pm 165$ & $558 \pm 233$ & $739 \pm 228$ \\
\hline MOD-FIT & $720 \pm 196$ & $633 \pm 273$ & $864 \pm 265$ \\
\hline PC-NLM (proposed recon) & $687 \pm 170$ & $570 \pm 247$ & $729 \pm 233$ \\
\hline
\end{tabular}

Signal cancellations in COMP and NLM-COMP result in overestimation of the ADC values, whereas noise accumulation in MAGN and NLM-MAGN leads to underestimation of the ADC values. MOD-FIT also overestimates ADC values, especially in $\mathrm{ROI}_{3}$ where the SNR is low. The proposed PC-NLM reconstruction prevents signal cancellations while preserving the accuracy of the ADC estimates.

1. A region with no phase issues and relatively high SNR,

2. a region with local phase issues, and

3. a region with relatively low SNR, but no visible phase issues.

Figure $8 \mathrm{C}$ shows the ADC maps generated by each method, and Table 1 lists the mean and SD of ADC values in each ROI and in each ADC map. The in vivo results listed in Table 1 are consistent with the trends in Figure 6 for the simulated DWI images. For example, in $\mathrm{ROI}_{1}$ where there are no phase issues, we expect COMP to provide the most accurate results. For that region, the proposed method yields ADC values that closely match those of COMP, whereas MAGN and NLM-MAGN underestimate and MOD-FIT overestimates the ADC values, as expected. In $\mathrm{ROI}_{2}$ where there are visible phase issues, COMP and NLM-COMP yield unreasonably high ADC values. Comparing these results to the simulation results in Figure 6B, one can presume that the true $\mathrm{ADC}$ is somewhere in between the results of MAGN and PC-NLM. Hence, we can deduce that MOD-FIT overestimates the ADC values for this ROI. In $\mathrm{ROI}_{3}$ where SNR is low but there are no visible phase issues, COMP, NLMMAGN, and PC-NLM yield similar ADC values. MOD-FIT and NLM-COMP give higher ADC values, while MAGN yields lower ADC values, as predicted by high NSR cases in $\mathrm{ROI}_{1}$ of Figure $6 \mathrm{~B}$. Importantly, the proposed method preserves the accuracy of the ADC maps while improving image quality, despite the presence of significant local phase errors in the acquisitions.

Figure 9 shows the results for the second subject, where DWI images and ADC maps are displayed for the case when diffusion-weighting was in the $\mathrm{A} / \mathrm{P}$ direction. Once again, COMP and NLM-COMP reconstructions suffer from clearly



FIGURE 9 Reconstructions from in vivo DTI acquisitions of the spinal cord for the second subject. Images with diffusion-encoding along the A/P direction are displayed. (A) DWI images are shown for the proposed PC-NLM and alternative reconstructions. Note the suppressed noise level and the lack of signal cancellations with the proposed reconstruction. (B) The corresponding ADC maps

visible signal cancellation (red arrows) near the discs, which in turn correspond to locations where nerve roots exit the cord. The noise accumulation problem in MAGN and NLMMAGN is especially prominent near the pons and in the inferior regions of the cervical spine. Although the results for MOD-FIT and PC-NLM match visually for the DWI image, the ADC map for MOD-FIT reveals overestimation problems in regions of low signal intensity. The proposed PC-NLM reconstruction provides visually improved image quality that is devoid of signal cancellations in these regions, with high fidelity ADC maps.

\section{5 | DISCUSSION}

High-resolution DWI is intrinsically SNR-starved. Although complex averaging of multiple acquisitions offers improved noise suppression when compared to magnitude averaging, phase errors among multiple acquisitions lead to signal cancellations in combined images. We observed that these signal cancellations are prominent in the spinal cord where substantial local motion is present along the $\mathrm{A} / \mathrm{P}$ direction. To the best of our knowledge, this local-motion-induced signal cancellation problem in the spinal cord has not been reported in the literature. Several factors might have contributed to this 
problem remaining elusive in clinical settings. First, DWI acquisitions are most commonly performed in the brain where local motion may not be as problematic as in the spinal cord. Second, the majority of DWI acquisitions are performed using ss-EPI followed by magnitude averaging across acquisitions. Last, even in the case of complex averaging, the described signal cancellation problem is encountered in a subset of the subjects that we have imaged during our studies. The relative infrequency of this problem makes it all the more important that it should be avoided, as signal cancellation may otherwise be mistaken for increased diffusion because of pathology.

The proposed reconstruction scheme incorporates a new PC-NLM filter that increases the image SNR via averaging across pixels with highly similar patches. Importantly, the search for similar patches takes into account both the magnitudes and the phases of the patch pixels. Although a few of the previous studies applied denoising on complex DWI data, they were restricted to single acquisitions (i.e., no repetitions to average). ${ }^{48,49}$ Here, the PC-NLM filter performs averaging to simultaneously suppress signal cancellations because of phase errors and reduce noise amplification. Our results demonstrate that when NSR $\geq 0.125$, the proposed reconstruction achieves higher PSNR and SSIM values than methods based on magnitude averaging. In addition, it yields comparable image quality to methods based on complex averaging, but without signal cancellation artifacts.

When compared to Rician noise model fitting, the proposed reconstruction has higher PSNR and SSIM for NSR $\geq 0.100$, while providing reliable ADC values even in low SNR regions. The problems of model fitting are exacerbated at low SNR cases and especially for fewer NEXs. For example, when the in vivo data from the first subject is reconstructed for $\mathrm{NEX}=8$ or NEX $=4$ (i.e., using only a subset of the 16 acquisitions), the performance of model fitting severely deteriorates (see Supporting Figure S6 for a comparison of all techniques for NEX $=8$ and NEX $=4$ ). This result is expected, as model fitting becomes increasingly challenging with fewer samples to fit. Another problem of model fitting is that it assumes the magnitude DWI images to follow a Rice distribution. In reality, however, both refocusing and partial $\mathrm{k}$-space reconstructions remove slowly varying phase information from images. In addition, partial $\mathrm{k}$-space reconstruction fills the missing half of $\mathrm{k}$-space using the acquired part. These processes cause the complex noise to deviate from a white Gaussian distribution, and thereby cause the magnitude DWI images to deviate from a Rice distribution. As such, in pixels where the signal and SD of noise are comparable, ML estimation can fail to find a nonnegative fit for the true signal intensity. Here, we assigned a small albeit non-zero value of $\sigma_{g} / 100$ to those pixels. Then, ADC can be under or overestimated depending on how the assigned value compares with the true signal intensity. Yet, this procedure is preferable to assigning a 0 value that would yield infinitely large ADC estimations. In contrast to these problems of model fitting, PC-NLM provides visually improved image quality even at reduced number of acquisitions, yielding reliable ADC estimates and successfully avoiding signal cancellation artifacts.

Signal cancellation artifacts because of motion-induced phase errors may also be mitigated to a certain extent with cardiac gating, if the cardiac synchronization is optimized to acquire the data at the periods of relative quiescence (e.g., starting $\sim 320 \mathrm{~ms}$ after the cardiac trigger signal). ${ }^{50}$ This approach may especially be useful for multi-shot acquisitions to alleviate phase inconsistencies among interleaved data, where the proposed method cannot be used in its current form. Although gating was not performed for the single-shot multiple-NEX in vivo data in this study, using a cardiac-gated acquisition can further increase the performance of the proposed reconstruction. It should be noted that cardiac-gating based approaches prolong the scan time considerably, especially when multiple averages are needed to increase the SNR. PC-NLM solves the signal cancellation issue while increasing the SNR, without the expense of prolonged scan time.

A potential limitation of the proposed reconstruction relates to the trade-off between noise suppression and spatial resolution, inherent to filtering. One way to mitigate resolution loss is to restrict the smoothing parameter to small values. Note that the smoothing parameter directly affects image quality, as well as the tolerance to magnitude differences and phase errors among neighborhoods. As the smoothing parameter increases, magnitude and phase differences become more tolerable, causing increased averaging and thereby blurring in the final image. To minimize potential losses in resolution, we compute the smoothing parameter before the POCS step that can amplify high frequency noise. We also set $\beta=0.5$, as typically done to minimize resolution loss in low-noise cases. ${ }^{20}$ When reconstructions were performed with $\beta=1$ instead, excessive image blurring occurred (results not shown). In future work, we plan to investigate the benefits of spatially adaptive selection of the smoothing parameter based on local SNR variations in the image (e.g., because of locally varying coil sensitivities). ${ }^{11}$ This approach could better preserve resolution in high-SNR regions like the pons or cerebellum, while successfully denoising regions with lower coil sensitivities such as the inferior cervical spine.

In spinal cord imaging, novel approaches that apply high $b$-value and/or axial acquisitions with $\mathrm{NEX}=1$ are emerging. ${ }^{46,51-54}$ Because PC-NLM targets multi-acquisition imaging, it does not have a direct application for NEX $=1$ cases. Nevertheless, the phase issues described in this work should inform the handling and acquisition of interleaved data. Note that ss-EPI with multiple acquisitions is still the most frequently used sequence in clinical settings. Although 
this work especially targeted that sequence, given its robustness against reduced SNR, we expect PC-NLM to perform equally well for high $b$-value imaging. For axial imaging of the spinal cord, we expect a potentially uniform phase over the cord, varying across acquisitions. Note that the phase over the cord would still be different than that of the background tissue, creating similar signal cancellation problems that might be overcome with the proposed PC-NLM reconstruction. In vivo demonstration of PC-NLM for non-sagittal orientations remains a future work.

In this work, we examined NLM filtering for spinal cord DWI unlike most of the prior work on NLM that focused on brain imaging. It could be hypothesized that morphological differences between the 2 anatomies might alter NLM's overall efficacy. Yet, we set the neighborhood size and search volume parameters for PC-NLM to $d=1$ and $M=5$ based on previously shown optimum values for NLM in the brain. ${ }^{20}$ When we tested different $d$ and $M$ values in our simulation framework for spinal cord DWI, we found that the image quality degrades with larger values of $d$, and the algorithm is nearly insensitive to changes in $M$ (results not shown). These observations fully agree with those in Coupe et al. ${ }^{20}$ The strong agreement between our observations and the reports in Coupe et al. ${ }^{20}$ could be attributed to a trade-off between the pixel count and signal heterogeneity. On the one hand, the pixel count is greater in the brain especially when compared to reduced-FOV spinal images. This potentially leads to a smaller number of similar patches to average in the spinal cord. On the other hand, the brain features a variety of different structures when compared to the homogeneous appearance of the spinal cord. In turn, the number of patches to average are increased in the spinal cord. Our results indicate that these 2 counteracting factors balance each other to yield consistent NLM performance in the brain and the spinal cord.

One of the in vivo results in this work incorporated a DTI data set, where the proposed method was demonstrated for only DWI along the A/P direction. For DTI and connectivity analyses, signal cancellations can alter the fractional anisotropy (FA) maps, which in turn can lead to incorrect estimation of the principle diffusion direction. In such cases, a modified version of the proposed reconstruction that takes into account the images from all diffusion directions may improve the quality of the FA maps. Although we have recently presented a preliminary analysis of this problem, ${ }^{55} \mathrm{a}$ detailed analysis that demonstrates the effects of the phase errors on the integrity of the FA maps and connectivity measures remains a future work.

\section{6 | CONCLUSIONS}

In this work, we have proposed a new PC-NLM reconstruction that reduces noise and suppresses signal cancellations because of motion-induced phase in DWI. This technique preserves the details of DWI images and improves image quality while yielding accurate ADC maps. Extensive simulations and in vivo DWI in the spinal cord of healthy subjects demonstrate the improved performance of the proposed technique over both magnitude averaging and complex averaging following global phase correction.

\section{ACKNOWLEDGMENTS}

This work was supported by the European Commission through an FP7 Marie Curie Career Integration Grant (PCIG13GA618834, PCIG13GA618101), by a European Molecular Biology Organization Installation Grant (IG 3028), by the Turkish Academy of Sciences through TUBA-GEBIP 2015 program, and by the BAGEP Award of the Science Academy

\section{REFERENCES}

[1] Dietrich O, Biffar A, Baur-Melnyk A, Reiser MF. Technical aspects of MR diffusion imaging of the body. Eur J Radiol. 2010;76:314-322.

[2] Le Bihan D, Mangin JF, Poupon C, Clark C, Pappata S, Molko N, Chabriat H. Diffusion tensor imaging: concepts and applications. J Magn Reson Imaging. 2001;13:534-546.

[3] Tournier JD, Mori S, Leemans A. Diffusion tensor imaging and beyond. Magn Reson Med. 2011;65:1532-1556.

[4] Bammer R, Fazekas F. Diffusion imaging of the human spinal cord and the vertebral column. Top Magn Reson Imaging. 2003; 14:461-476.

[5] Anderson AW, Gore JC. Analysis and correction of motion artifacts in diffusion weighted imaging. Magn Reson Med. 1994;32: 379-387.

[6] Dietrich O, Heiland S, Sartor K. Noise correction for the exact determination of apparent diffusion coefficients at low SNR. Magn Reson Med. 2001;45:448-453.

[7] Lam F, Babacan SD, Haldar JP, Weiner MW, Schuff N, Liang ZP. Denoising diffusion-weighted magnitude MR images using rank and edge constraints. Magn Reson Med. 2014;71:1272-1284.

[8] Kristoffersen A. Diffusion measurements and diffusion tensor imaging with noisy magnitude data. J Magn Reson Imaging. 2009;29:237-241.

[9] Descoteaux M, Wiest-Daesslé N, Prima S, Barillot C, Deriche R. Impact of Rician adapted non-local means filtering on HARDI. Med Image Comput Comput Assist Interv. 2008;11:122-130.

[10] Manjon JV, Thacker NA, Lull JJ, Garcia-Marti G, Marti-Bonmati L, Robles M. Multicomponent MR image denoising. Int J Biomed Imaging. 2009;2009:756897.

[11] Manjon JV, Coupe P, Marti-Bonmati L, Collins DL, Robles M. Adaptive non-local means denoising of MR images with spatially varying noise levels. J Magn Reson Imaging. 2010;31:192-203.

[12] Wiest-Daesslé N, Prima S, Coupé P, Morrissey SP, Barillot C. Rician noise removal by non-local means filtering for low signal-to-noise ratio MRI: applications to DT-MRI. Med Image Comput Comput Assist Interv. 2008;11:171-179. 
[13] Basu S, Fletcher T, Whitaker R. Rician noise removal in diffusion tensor MRI. Med Image Comput Comput Assist Interv. 2006;9:117-125.

[14] Aja-Fernandez S, Niethammer M, Kubicki M, Shenton ME, Westin CF. Restoration of DWI data using a Rician LMMSE estimator. IEEE Trans Med Imaging. 2008;27:1389-1403.

[15] Aja-Fernandez S, Alberola-Lopez C, Westin CF. Noise and signal estimation in magnitude MRI and Rician distributed images: a LMMSE approach. IEEE Trans Image Process. 2008;17:13831398.

[16] Tristan-Vega A, Aja-Fernandez S. DWI filtering using joint information for DTI and HARDI. Med Image Anal. 2010;14: 205-218

[17] Krissian K, Aja-Fernandez S. Noise-driven anisotropic diffusion filtering of MRI. IEEE Trans Image Process. 2009;18:2265-2274.

[18] Ding Z, Gore JC, Anderson AW. Reduction of noise in diffusion tensor images using anisotropic smoothing. Magn Reson Med. 2005;53:485-490.

[19] McGraw T, Vemuri B, Özarslan E, Chen Y, Mareci T. Variational denoising of diffusion weighted MRI. Inverse Probl Imag. 2009;3:625-648.

[20] Coupe P, Yger P, Prima S, Hellier P, Kervrann C. An optimized blockwise non local means denoising filter for 3D magnetic resonance images. IEEE Trans Med Imaging. 2007;27:425-441.

[21] Nowak RD. Wavelet-based Rician noise removal for magnetic resonance imaging. IEEE Trans Image Process. 1999;8:14081419.

[22] Miller KL, Pauly JM. Nonlinear phase correction for navigated diffusion imaging. Magn Reson Med. 2003;50:343-353.

[23] Pipe JG, Farthing VG, Forbes KP. Multishot diffusion-weighted FSE using PROPELLER MRI. Magn Reson Med. 2002;47:42-52.

[24] Aksoy M, Skare S, Holdsworth S, Bammer R. Effects of motion and b-matrix correction for high resolution DTI with short-axis PROPELLER-EPI. NMR Biomed. 2010;23:794-802.

[25] Porter DA, Heidemann RM. High resolution diffusion-weighted imaging using readout-segmented echo-planar imaging, parallel imaging and a two-dimensional navigator-based reacquisition. Magn Reson Med. 2009;62:468-475.

[26] Liu C, Moseley ME, Bammer R. Simultaneous phase correction and SENSE reconstruction for navigated multi-shot DWI with non-cartesian k-space sampling. Magn Reson Med. 2005;54: 1412-1422.

[27] Aksoy M, Forman C, Straka M, Skare S, Holdsworth S, Hornegger J, Bammer R. Real-time optical motion correction for diffusion tensor imaging. Magn Reson Med. 2011;66:366-378.

[28] Aksoy M, Forman C, Straka M, Çukur T, Hornegger J, Bammer R. Hybrid prospective and retrospective head motion correction to mitigate cross-calibration errors. Magn Reson Med. 2012;67: 1237-1251.

[29] Herbst M, MacLaren J, Weigel M, Korvink J, Hennig J, Zaitsev M. Prospective motion correction with continuous gradient updates in diffusion weighted imaging. Magn Reson Med. 2012; 67:326-338.

[30] Figley CR, Stroman PW. Investigation of human cervical and upper thoracic spinal cord motion: implications for imaging spinal cord structure and function. Magn Reson Med. 2007;58:185-189.
[31] Matsuzaki H, Wakabayashi K, Ishihara K, Ishikawa H, Kawabata $\mathrm{H}$, Onomura T. The origin and significance of spinal cord pulsation. Spinal Cord. 1996;34:422-426.

[32] Winklhofer S, Schoth F, Stolzmann P, Krings T, Mull M, Wiesmann M, Stracke CP. Spinal cord motion: influence of respiration and cardiac cycle. Rofo. 2014;186:1016-1021.

[33] Zhong X, Meyer CH, Schlesinger DJ, Sheehan JP, Epstein FH, Larner JM, Benedict SH, Read PW, Sheng K, Cai J. Tracking brain motion during the cardiac cycle using spiral cine-DENSE MRI. Med Phys. 2009;36:3413-3419.

[34] Buades A, Coll B, Morel JM. A review of image denoising algorithms, with a new one. Multiscale Model Simul. 2006;66:1383-1406.

[35] Gasser T, Sroka L, Christine JS. Residual variance and residual pattern in nonlinear regression. Biometrika. 1986;73:625-633.

[36] Boulanger J, Kervrann C, Bouthemy P. Adaptive spatio-temporal restoration for 4D fluorescence microscopic imaging. Med Image Comput Comput Assist Interv. 2005;8:893-901.

[37] Haacke EM, Lindskogj ED, Lin W. A fast, iterative, partialfourier technique capable of local phase recovery. J Magn Reson. 1991;92:126-145.

[38] De Leener B, Cohen-Adad J, Kadoury S. Automatic segmentation of the spinal cord and spinal canal coupled with vertebral labeling. IEEE Trans Med Imaging. 2015;34:1705-1718.

[39] Fonov VS, Le Troter A, Taso M, et al. Framework for integrated MRI average of the spinal cord white and gray matter: the MNIPoly-AMU template. Neuroimage. 2014;102:817-827.

[40] Helenius J, Soinne L, Perkiö J, Salonen O, Kangasmäki A, Kaste M, Carano RAD, Aronen HJ, Tatlisumak T. Diffusion-weighted MR imaging in normal human brains in various age groups. Am J Neuroradiol. 2002;23:194-199.

[41] Clark CA, Barker GJ, Tofts PS. Magnetic resonance diffusion imaging of the human cervical spinal cord in vivo. In Proceedings of the 6th Annual Meeting of ISMRM, Sydney, Australia, 1998. p. 529.

[42] Saritas EU, Cunningham CH, Lee JH, Han ET, Nishimura DG. DWI of the spinal cord with reduced FOV single-shot EPI. Magn Reson Med. 2008;60:468-473.

[43] Saritas EU, Lee JH, Nishimura DG. SNR dependence of optimal parameters for apparent diffusion coefficient measurements. IEEE Trans Med Imaging. 2011;30:424-437.

[44] Gudbjartsson H, Patz S. The Rician distribution of noisy MRI data. Magn Reson Med. 1995;34:910-914.

[45] Koay CG, Basser PJ. Analytically exact correction scheme for signal extraction from noisy magnitude MR signals. J Magn Reson. 2006;179:317-322.

[46] Grussu F, Schneider T, Zhang H, Alexander DC, Wheeler-Kingshott CAM. Neurite orientation dispersion and density imaging of the healthy cervical spinal cord in vivo. Neuroimage. 2015; 111:590-601.

[47] Wang Z, Bovik AC, Sheikh HR, Simoncelli EP. Image quality assessment: from error visibility to structural similarity. IEEE Trans Image Process. 2004;13:600-612.

[48] Wirestam R, Bibic A, Lätt J, Brockstedt S, Ståhlberg F. Denoising of complex MRI data by wavelet-domain filtering: application to high-b-value diffusion-weighted imaging. Magn Reson Med. 2006;56:1114-1120. 
[49] Haldar JP, Wedeen VJ, Nezamzadeh M, Dai G, Weiner MW, Schuff $\mathrm{N}$, Liang ZP. Improved diffusion imaging through SNR-enhancing joint reconstruction. Magn Reson Med. 2013;69:277-289.

[50] Summers P, Staempfli P, Jaermann T, Kwiecinski S, Kollias S. A preliminary study of the effects of trigger timing on diffusion tensor imaging of the human spinal cord. AJNR Am J Neuroradiol. 2006;27:1952-1961.

[51] By S, Xu J, Box BA, Bagnato FR, Smith SA. Application and evaluation of NODDI in the cervical spinal cord of multiple sclerosis patients. Neuroimage Clin. 2017;15:333-342.

[52] Duval T, Le Vy S, Stikov N, et al. g-Ratio weighted imaging of the human spinal cord in vivo. Neuroimage. 2017;145:11-23.

[53] Duval T, McNab JA, Setsompop K, Witzel T, Schneider T, Huang SY, Keil B, Klawiter EC, Wald LL, Cohen-Adad J. In vivo mapping of human spinal cord microstructure at $300 \mathrm{mT} / \mathrm{m}$. Neuroimage. 2015;118:494-507.

[54] Farrell JAD, Smith SA, Gordon-Lipkin EM, Reich DS, Calabresi PA, Van Zijl PCM. High b-value q-space diffusion-weighted MRI of the human cervical spinal cord in vivo: feasibility and application to multiple sclerosis. Magn Reson Med. 2008;59: 1079-1089.

[55] Kafali SG, Cukur T, Saritas EU. Joint non-local means reconstruction for correction of phase-induced errors in diffusion tensor imaging. In Proceedings of the 25th Annual Meeting of ISMRM, Honolulu, HI, 2017. p.7364.

\section{SUPPORTING INFORMATION}

Additional Supporting Information may be found online in the supporting information tab for this article.

FIGURE S1 Phase images from each acquisition for the case when diffusion-encoding is along the A/P direction. Four out of 16 acquisitions are displayed explicitly. For each acquisition, phase images from 4 channels out of 6 are displayed following a refocusing reconstruction to correct for global phase errors and a partial k-space reconstruction (the remaining 2 channels are not shown as they contained noise only). These local phase problems occur at exactly the same position for images obtained from all receive channels, indicating that they in fact stem from anatomical sources.

FIGURE S2 Local phase problems in DWI of the cervical spine for different diffusion-encoding directions. (A, C, E) Magnitude and phase of each acquisition when diffusionencoding is along $\mathrm{A} / \mathrm{P}, \mathrm{S} / \mathrm{I}$, and $\mathrm{R} / \mathrm{L}$ directions, displayed following a global phase correction and a partial k-space reconstruction. (B, D, F) The results of complex averaging (COMP) and magnitude averaging (MAGN) across multiple acquisitions. MAGN results in noise accumulation for all diffusion encoding directions. COMP, on the other hand, suffers from signal cancellations because of local phase problems (shown by red arrows), which are prominent when the diffusion-encoding is along the A/P direction. These phase problems occur at the positions where the nerve roots exit the spinal cord, which is consistent with the literature on nerve root pulsations in the same direction, synchronized to heart and CSF pulsations.

FIGURE S3 Diffusion-weighted images from DTI of the spinal cord in 12 different directions. The results are shown for complex averaging (COMP) and magnitude averaging (MAGN). The vectors on top of the images indicate the direction of diffusion encoding: [S/I A/P R/L], respectively. We observed that whereas local signal cancellations are visible in many of the images, they are most prominent when the diffusion-encoding direction has a significant component along the A/P direction (e.g., $\left[\begin{array}{lll}0 & 1 & 0\end{array}\right]$ case displayed in red box).

FIGURE S4 DWI simulation results at NSR $=0.250$ for the case of global phase only and the case of both global and local phase errors. (A) Magnitude and phase of each of the acquisitions for the simulated data without any local phase errors, displayed following a global phase correction and a partial k-space reconstruction. (B) Results of alternative reconstructions and the proposed method are depicted. Note the suppressed noise in the proposed reconstruction when compared to MAGN and NLM-MAGN. Because there are no local phase errors, COMP and NLM-COMP do not show any signal cancellations. NLM-COMP improves image SNR when compared to COMP. MODFIT results in an unnatural look, especially in low signal intensity regions. The proposed method has comparable image quality to that of NLM-COMP. (C) Magnitude and phase of each of the acquisitions for the simulated data with both global and local phase errors, displayed following a global phase correction and partial k-space reconstruction. Phase errors, shown by red arrows, are added to the DWI images with the diffusion-encoding along the A/P direction. (D) Results of alternative reconstructions and the proposed method are depicted. Signal cancellations because of phase errors are prominent in both COMP and NLMCOMP. Likewise, high-level noise causes noise accumulation in MAGN and the preceding NLM filter is not sufficient to overcome this issue in NLM-MAGN. The performance of MOD-FIT is almost unchanged, with a darkening in the background regions. On the other hand, the proposed PC-NLM renders a solution without any phase cancellations while suppressing noise.

FIGURE S5 Local noise statistics for all reconstruction techniques at NSR $=0.250$, for the case of global phase only and the case of both global and local phase errors. (A) For global-phase-only case, COMP and MAGN result in comparable high SD. MOD-FIT has the highest standard deviation among all reconstruction techniques, because of its noise sensitivity. NLM-COMP and NLM-MAGN have relatively reduced SD when compared to their non-filtered counterparts. PC-NLM yields the lowest SD, demonstrating robustness against noise. (B) For the case of both global 
and local phase errors, the noise statistics are almost unchanged for MAGN, NLM-MAGN, and MOD-FIT, as expected. In regions of signal cancellations, SD for COMP and NLM-COMP are reduced because of lower pixel intensities in those regions. PC-NLM yields the lowest SD in regions without signal cancellations. In cancellation regions, SD of PC-NLM is slightly lower than that of MAGN. The noise statistics in 3 different ROIs (marked over the COMP image in A) are listed in Supporting Table S1.

FIGURE S6 Results for in vivo DWI of the spinal cord for the first subject for (A) $\mathrm{NEX}=8$ and (B) $\mathrm{NEX}=4$ (i.e., using only a subset of the 16 acquisitions). As NEX is reduced, the image qualities for COMP, NLM-COMP, and MOD-FIT significantly deteriorate. The number and extent of signal cancellation regions are increased for COMP and NLM-COMP, as the likelihood of having a NEX with similar phase is reduced when there are fewer NEXs. The image quality for MOD-FIT especially suffers in inferior parts of the spine (shown by blue arrows) because of reduced coil sensitivities. This problem is further exacerbated as NEX is reduced, as model fitting becomes more challenging when there are fewer samples for a low-SNR pixel. MAGN, NLM-MAGN, and PC-NLM show lower SNR with reduced NEX, but the image qualities are not affected otherwise. Overall, when compared to the other reconstruction techniques, PC-NLM has visibly improved image quality at both $\mathrm{NEX}=8$ and $\mathrm{NEX}=4$, and it does not suffer from local signal cancellations or noise accumulation problems.

TABLE S1 Noise statistics in 3 different ROIs for the results in Supporting Figure S5.

How to cite this article: Kafali SG, Çukur T, Saritas EU. Phase-correcting non-local means filtering for diffusion-weighted imaging of the spinal cord. Magn Reson Med. 2018;80:1020-1035. https://doi.org/ $\underline{10.1002 / \mathrm{mrm} .27105}$ 